\title{
DISTROFIA MACULAR ANULAR BENIGNA CONCÉNTRICA
}

\section{BENIGN CONCENTRIC ANNULAR MACULAR DYSTROPHY}

\author{
GÓMEZ-FAIÑA $\mathrm{P}^{1}$, ALARCÓN-VALERO I ${ }^{1}$, BUIL CALVO JA ${ }^{2}$, CALSINA-PRAT M ${ }^{1}$, \\ MARTÍN-MORAL D ${ }^{1}$, LILLO-SOPENA J ${ }^{3}$, CASTILLA CÉSPEDES M${ }^{4}$
}

\section{RESUMEN}

Caso clínico: Presentamos un caso clínico de distrofia macular anular benigna concéntrica. Se trata de una mujer de 32 años que se presenta con pérdida de calidad visual. Se realiza exploración oftalmoscópica, angiografía fluoresceínica, pruebas electrofisiológicas y campimetría.

Discusión: Es de gran importancia realizar un cuidadoso diagnóstico diferencial con otras distrofias que presenten un patrón angiográfico en «ojo de buey», dado que la conservación de una relativamente buena agudeza visual es una característica señalada de esta enfermedad.

Palabras clave: Distrofia macular anular benigna concéntrica, maculopatía en «ojo de buey», angiografía fluoresceínica, pruebas electrofisiológicas, gen IMPG1.

\begin{abstract}
Case report: A case of benign concentric annular macular dystrophy is described. A 32-year-old woman presented with loss of quality in visual acuity. Ophthalmologic examination, fluorescein angiogram, electrophysiologic tests and visual field measurements were performed.

Discussion: It is very important to include in the differential diagnosis other dystrophies which present a «bull's eye» pattern on fluorescein angiography, given that preservation of relatively good visual acuity is a special feature of this disease (Arch Soc Esp Oftalmol 2007; 82: 373-376).
\end{abstract}

Key words: Benign concentric annular macular dystrophy, «bull's eye» maculopathy, fluorescein angiography, electrophysiological tests, IMPG1 gene.

\footnotetext{
Recibido: 9/12/05. Aceptado: 18/4/07.

2 Doctor en Medicina. Hospital de San Pablo.

3 Licenciado en Medicina. Hospital de Bellvitge.

Correspondencia:

Pablo Gómez Faiña

C/. Gran Vía Carlos III, 95, 5. ${ }^{\circ}$ 1. $^{\mathrm{a}}$ esc. B

08028 Barcelona

España

E-mail: 36836pgf@comb.es
}

Hospital de la Esperanza y Hospital del Mar, Universidad Autónoma de Barcelona. Barcelona. Hospital de San Pablo, Universidad Autónoma de Barcelona. Barcelona. Hospital de Bellvitge, Hospitalet de Llobregat, Universidad de Barcelona. Barcelona. España.

1 Licenciado en Medicina. Hospital de la Esperanza y Hospital del Mar.

4 Doctor en Medicina. Hospital de la Esperanza y Hospital del Mar.

Comunicación presentada en el LXXXI Congreso de la S.E.O. (Zaragoza 2005). 


\section{INTRODUCCIÓN}

La distrofia macular anular benigna concéntrica es una entidad poco frecuente, que fue individualizada por Deutman en 1974 (1) como un trastorno de herencia autosómica dominante. La agudeza visual normalmente se encuentra bien conservada y en el fondo de ojo se puede observar un defecto concéntrico y bilateral del epitelio pigmentario macular, cuya traducción angiográfica es una hiperfluorescencia en forma de anillo. También pueden existir alteraciones campimétricas, electrorretinográficas y de la visión de los colores.

Recientemente se ha asociado la distrofia macular anular benigna concéntrica a una aparente mutación del gen IMPG1 (interphotoreceptor matrix proteoglycan 1), que reside en el cromosoma 6.

\section{CASO CLÍNICO}

Mujer de 32 años, sin antecedentes de interés, que refiere pérdida de calidad visual durante el día. $\mathrm{Su}$ agudeza visual sin corrección óptica es de 1 con ambos ojos. En la exploración del polo posterior, observamos en ambos ojos una alteración del epitelio pigmentario retiniano (EPR) en la región macular y acúmulos pigmentarios aislados en retina periférica (figs. 1 y 2). La perimetría computarizada Humphrey 30-2 muestra un escotoma paracentral en ambos ojos (figs. 3 y 4). En la angiografía fluoresceínica $(\mathrm{AGF})$ se observa una hiperfluorescencia

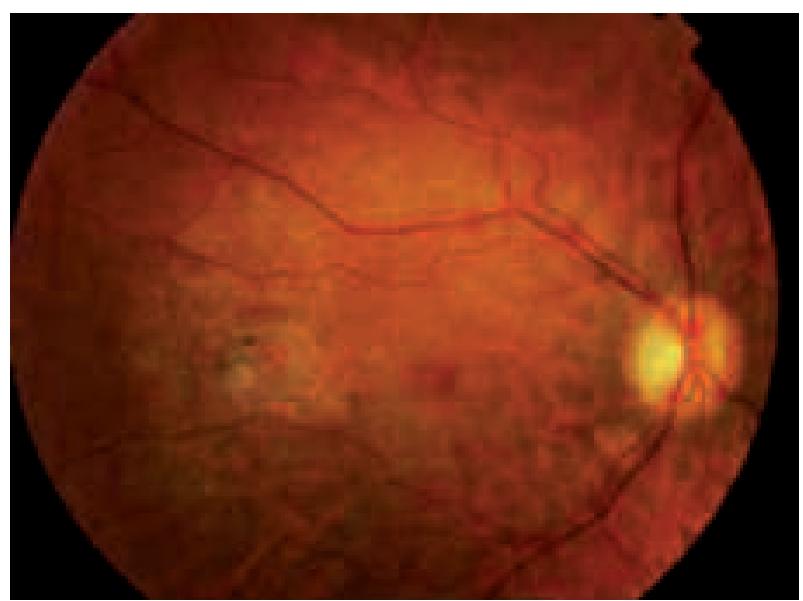

Fig. 1: Retinografía ojo derecho: alteración del epitelio pigmentario retiniano en la región macular y acúmulos pigmentarios aislados en retina periférica.

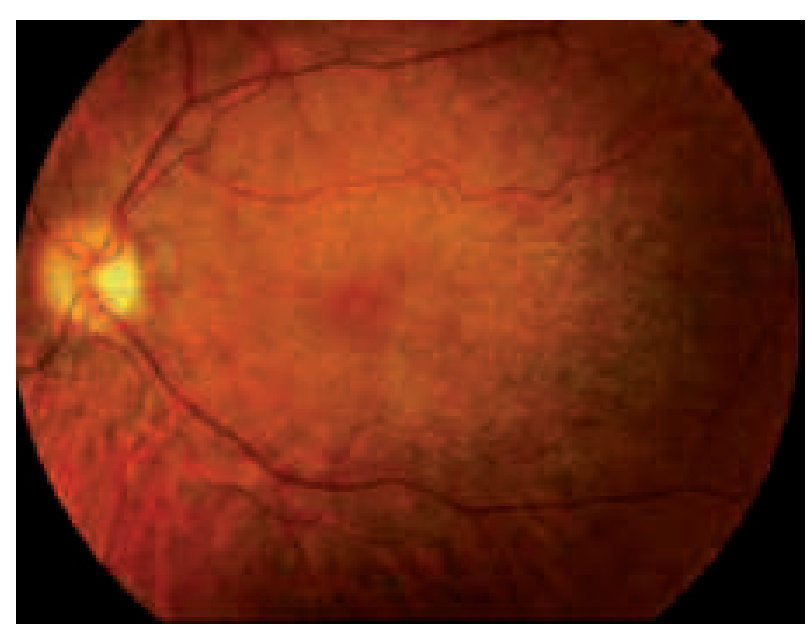

Fig. 2: Retinografía ojo izquierdo: alteración del epitelio pigmentario retiniano en la región macular y acúmulos pigmentarios aislados en retina periférica.

bilateral por efecto ventana compatible con imagen angiográfica en «ojo de buey» en ambos ojos (figs. 5 y 6). El test de visión cromática fue normal en ambos ojos. Las pruebas electrofisiológicas realizadas, electrorretinograma (ERG) y electrooculograma (EOG), no mostraron alteraciones. Se ha realizado el seguimiento de la paciente sin que se hayan

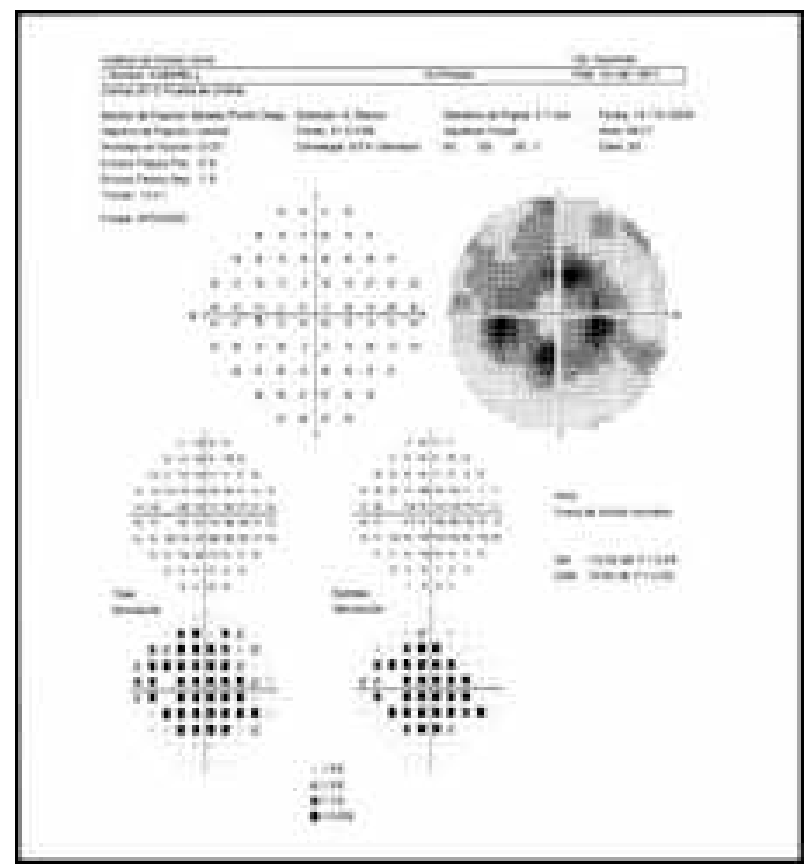

Fig. 3: Perimetría computarizada Humphrey 30-2 ojo derecho: escotoma paracentral. 


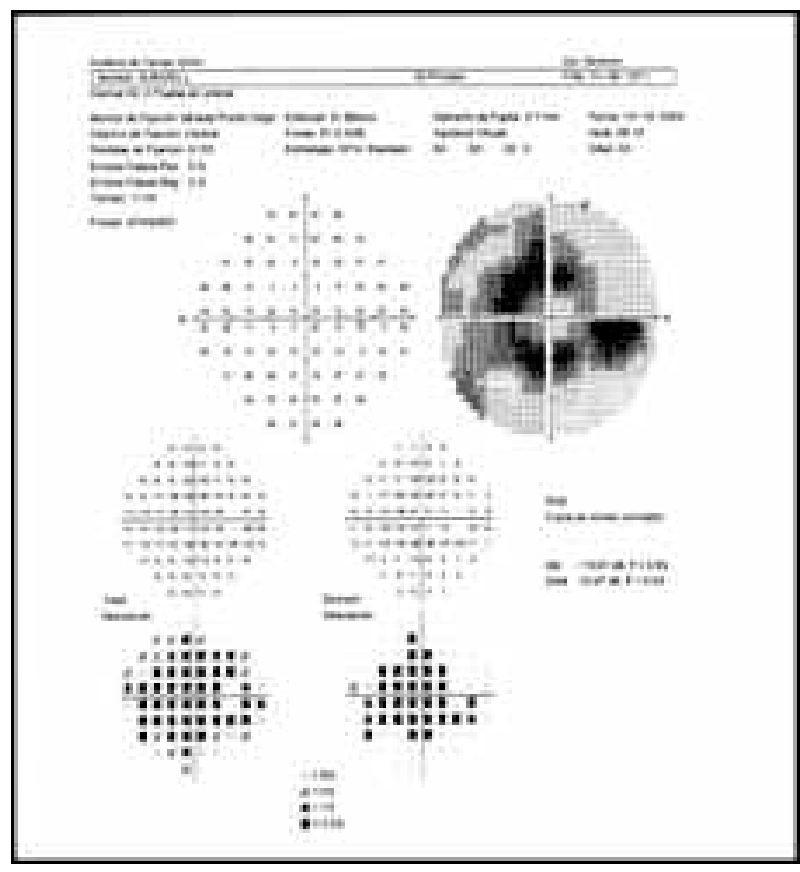

Fig. 4: Perimetría computarizada Humphrey 30-2 ojo izquierdo: escotoma paracentral.

objetivado cambios en las distintas pruebas exploratorias realizadas hasta el momento.

\section{DISCUSIÓN}

La distrofia macular anular benigna concéntrica posee una herencia autosómica dominante y una expresión clínica muy variable (1). Su signo más

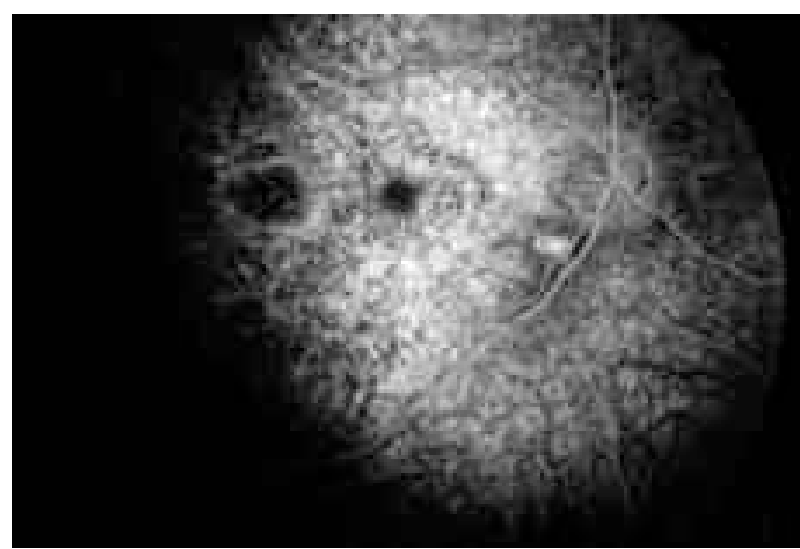

Fig. 5: Angiografía fluoresceínica ojo derecho: hiperfluorescencia por efecto ventana compatible con imagen angiográfica en «ojo de buey».

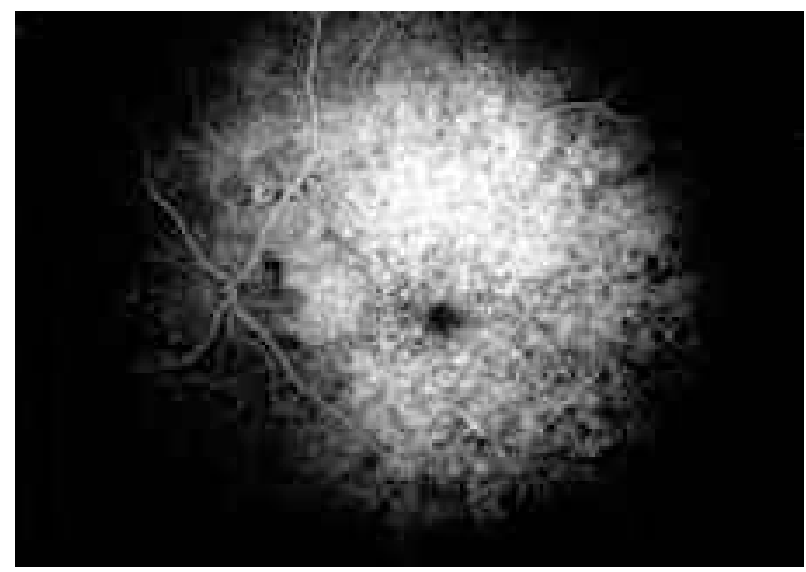

Fig. 6: Angiografía fluoresceínica ojo izquierdo: hiperfluorescencia por efecto ventana compatible con imagen angiográfica en «ojo de buey».

característico es la alteración concéntrica del EPR de la región macular, cuya traducción angiográfica es una hiperfluorescencia de morfología circular debida al efecto ventana correspondiente al área de atrofia de EPR, proporcionando la característica imagen de «mácula en ojo de buey». La conservación de una relativamente buena agudeza visual es una característica señalada de esta enfermedad. El defecto campimétrico más comúnmente descrito son los escotomas centrales y paracentrales, que para poder diferenciar con exactitud necesitarán de una perimetría de campo amplio. En cuanto a la visión de los colores predomina, aunque no es constante, la existencia de defectos en el eje azul-amari1lo (1).

El perfil electrofisiológico de esta distrofia es muy variable, pudiendo ser normales ERG y EOG, como en nuestro caso, lo que sugiere un defecto focal macular más que un defecto generalizado. Debido a la disfunción no selectiva de conos y bastones, es tentador el localizar el defecto primario de esta enfermedad en el epitelio pigmentario retiniano (2).

La imagen angiográfica de «mácula en ojo de buey», los antecedentes familiares y el patrón hereditario, constituyen los datos más relevantes para establecer el diagnóstico diferencial entre diversas entidades clínicas (1). La retinopatía por antipalúdicos de síntesis precisa el antecedente de ingesta de cloroquina o hidroxicloroquina. En la distrofia de conos (patrón hereditario heterogéneo; dominante, recesivo o ligado al cromosoma $\mathrm{X}$ ), algunos síntomas y signos clínicos como fotofobia, defecto seve- 
ro de la visión de los colores y sobre todo un electrorretinograma fotópico muy alterado, ayudan al diagnóstico. La enfermedad de Stargardt (patrón hereditario autonómico recesivo generalmente), manifiesta desde una edad temprana una marcada disminución de la agudeza visual, aunque en fases iniciales puede tener fondo de ojo normal a pesar de la pérdida severa de agudeza visual, pero el diagnóstico diferencial se establece con el silencio coroideo angiográfico característico y el EOG alterado (2). La distrofia macular fenestrada brillante, presenta unos brillos característicos (3) y la distrofia areolar central (patrón hereditario autonómico dominante) cursa con buena agudeza visual inicial y empeora con la aparición de atrofia de bordes bien delimitados sin patrón anular y con afectación central (3). La retinitis pigmentosa inversa presenta importante afectación incial de ERG y EOG $(2,3)$.

Es importante, de cara al minucioso diagnóstico diferencial, hacer referencia a los antecedentes familiares mostrando especial relevancia la exploración oftalmológica de los padres, ya que al tratarse de una enfermedad genética que posee una herencia autosómica dominante, salvo mutaciones «de novo», cabría esperar que alguno de los progenitores, aunque asintomáticos, presentasen signos de la enfermedad. Destacar también la ayuda que podría suponer en el futuro para el diagnóstico diferencial, el estudio genético molecular (4).
Debido al relativo buen pronóstico de esta distrofia, es necesario realizar un diagnóstico preciso que permita distinguirla de otras entidades, para ello son fundamentales las pruebas electrofisiológicas (ERG y EOG) y la AFG (3).

Aunque el término «benigno» fue aplicado por Deutman debido a que la visión no se modificaba, posteriormente se observó que algunos casos evolucionaban a una atrofia más generalizada con afectación de conos y bastones, disminución progresiva de la agudeza visual y discromatopsia $(2,5)$.

\section{BIBLIOGRAFÍA}

1. Deutman AF. Benign concentric annular macular dystrophy. Am J Ophthalmol 1974; 78: 384-396.

2. van den Biesen PR, Deutman AF, Pinckers AJ. Evolution of benign concentric annular macular dystrophy. Am J Ophthalmol 1985; 100: 73-78.

3. Pérez Álvarez MJ, Clement Fernández F. Distrofia macular anular benigna concéntrica: dos casos. Arch Soc Esp Oftalmol 2003; 78: 451-454.

4. van Lith-Verhoeven JJ, Hoyng $C B$, van den Helm B, Deutman AF, Brink HM, Kemperman $M H$, et al. The benign concentric annular macular dystrophy locus maps to 6p12.3-q16. Invest Ophthalmol Vis Sci 2004; 45: 3035 .

5. Salinas Alamán A, Sádaba Echarri LM, Corcóstegui Crespo I, García Layana A. Distrofia macular anular benigna. Arch Soc Esp Oftalmol 2005; 80: 45-48. 